\title{
Domain walls in antiferromagnets: The effect of Dzyaloshinskii-Moriya interactions
}

Cite as: J. Appl. Phys. 127, 223908 (2020); doi: 10.1063/5.0009409

Submitted: 30 March 2020 . Accepted: 22 May 2020 .

Published Online: 9 June 2020

Teo Conzelmann, Severin Selzer, (i) and Ulrich Nowak ${ }^{\text {a) (D) }}$

AFFILIATIONS

Department of Physics, University of Konstanz, D-78457 Konstanz, Germany

Note: This paper is part of the special topic on Antiferromagnetic Spintronics.

a)Author to whom correspondence should be addressed: ulrich.nowak@uni-konstanz.de

\begin{abstract}
We investigate domain walls in antiferromagnets focusing on the effect of Dzyaloshinskii-Moriya interactions (DMIs). Using spin model simulations and analytical arguments within a continuum theory, we show that Dzyaloshinskii-Moriya interactions affect static as well as dynamic properties of the domain wall. For certain configurations of the DMI vectors, the DMI can either tilt the easy plane of the domain wall, an effect that leads to a reduced domain wall width, or it can favor a certain chirality of the domain wall. Depending on the DMI configuration, the DMI may lead to an increasing or decreasing domain wall velocity. The asymmetry of the domain wall velocity observed in ferromagnets subject to DMI cannot be found in antiferromagnetic systems.
\end{abstract}

Published under license by AIP Publishing. https://doi.org/10.1063/5.0009409

\section{INTRODUCTION}

Conventional spintronic devices are mostly based on ferromagnetic materials or on heterostructures comprised of different types of materials, such as ferromagnetic/antiferromagnetic bilayers, to achieve an exchange bias. ${ }^{1,2}$ However, recent advances in writing and reading magnetic states of antiferromagnets (AFMs) have paved the way for pure antiferromagnetic spintronics. ${ }^{3-6}$ Possible advantages of AFMs for spintronics applications include their lack of stray fields ${ }^{4,7}$ and the rich choice of new materials, including a variety of antiferromagnetic insulators. ${ }^{6}$ The very low susceptibility to external fields, which used to be the biggest obstacle to applications, can be overcome by writing domains with spin-transfer or spin-orbit torques. Moreover, AFMs are found to have intrinsic time scale orders of magnitude shorter than those of ferromagnets (FMs). ${ }^{9-11}$ While this is an advantage for the control of magnetic states on ultrashort time scales, it was recently shown to be a disadvantage for the thermal stability of antiferromagnetic nanostructures. ${ }^{12}$

The stability problem can possibly be overcome following Parkin's idea of revising digital storage by using domain walls (DWs) to encode bits instead of particle-based domain patterns. ${ }^{13}$ Ferromagnetic DWs can be driven by external fields, ${ }^{14,15}$ spinpolarized currents, ${ }^{16-18}$ and temperature gradients. ${ }^{19,20}$ The latter is especially interesting since this effect should appear in AFMs as well as in FMs. The basic understanding of DW motion in temperature gradients rests on the fundamental thermodynamic potential for a domain wall, its free energy density, which is a monotonously decaying function of temperature, vanishing at the Curie temperature. Assuming a temperature gradient along the spatial coordinate of a nanowire, the minimization of free energy leads to an entropic torque acting on the domain wall that drags it toward the hotter region. These purely thermodynamic considerations are accompanied by a microscopic interpretation, where the space dependent thermal excitation leads to a spin wave current since the hotter region of a nanowire contains a higher density of magnons which, by diffusive motion, move into the cooler region. Assuming conservation of angular momentum, the magnonic spin current that passes the wall must push it toward the hotter region, a result that agrees qualitatively with the former thermodynamic argument.

Surprising effects were found for ferrimagnets. ${ }^{21,22}$ While below the Walker breakdown where the wall leaves its easy plane and starts precessing slowing down the DW motion, ${ }^{14}$ the temperature gradient always pulls the domain wall toward the hotter region above the Walker breakdown, and the ferrimagnetic DW can show the opposite, counterintuitive behavior of moving toward the cold end. In this case, the motion to either the hotter or the colder ends is driven by angular momentum transfer and, therefore, strongly affected by the angular momentum compensation temperature, a unique property of ferrimagnets. ${ }^{21}$ 
For domain walls in antiferromagnetic materials, ${ }^{23}$ recent publications forecast a variety of surprising effects, such as, e.g., a sign change of the domain wall velocity. ${ }^{24,25}$ However, this sign change can only appear for monochromatic excitation of spin waves with unique polarization but not for thermally excited spin waves. ${ }^{26}$ Nevertheless, a striking difference to the dynamics of domain walls in ferromagnets was found recently. It was shown that the Walker breakdown is missing in antiferromagnets. ${ }^{26}$ This is due to the fact that the antiferromagnetic wall is not tilted during its motion because the torques that are responsible for the tilting of the two sublattices of the antiferromagnet cancel each other. For the same reason, antiferromagnetic DWs have no inertia related to this tilting and, hence, no acceleration phase, leading to higher effective mobility. ${ }^{9,26}$ Similarly, significantly enhanced DW velocities and a deferred Walker breakdown have been reported for current induced DW motion in synthetic AFMs, where the antiferromagnetic coupling between the two ferromagnetic layers stabilizes the domain wall structure. ${ }^{27,28}$

The progress in controlling magnetic states in AFMs is due to the fact that staggered spin transfer torques can be observed in AFMs with broken inversion symmetry. Systems with artificially or intrinsically broken inversion symmetry have recently attracted much of interest because of their connection to topology. In systems with broken inversion symmetry, the Dzyaloshinskii-Moriya interaction (DMI) can be observed, ${ }^{29-31}$ a relativistic interaction which results from spin-orbit coupling and favors non-parallel alignment of neighboring spins, leading, e.g., to the formation of spin spirals ${ }^{32}$ or topologically non-trivial spin structures like skyrmions. ${ }^{33-35}$ The influence of the DMI on DW motion in FMs has already been investigated in several studies. In field-driven DWs, the Walker breakdown can be suppressed by $\mathrm{DMI}^{36}$ and in magnon-driven DWs it is possible to achieve higher velocities due to DMI. ${ }^{37}$ Furthermore, it was observed that field-driven DWs propagate asymmetrically ${ }^{38}$ since DMI breaks the symmetry of the dispersion relation. ${ }^{39,40}$

However, the influence of DMI on DWs in AFMs has not been investigated so far and it is the purpose of this work to explore thermally induced DW motion in AFMs theoretically, with a particular focus on the symmetry between the sublattices, which is key for a fast and efficient driving of antiferromagnetic DWs. We will compare analytical calculations in terms of a one-dimensional continuum model to numerical data based on atomistic spin dynamics simulations.

\section{SPIN MODEL}

The DW is modeled within the framework of an atomistic spin model consisting of classical spins $S_{i}$ on a simple cubic lattice with lattice constant $a$ described by the Hamiltonian,

$$
\mathcal{H}=-J \sum_{\langle i, j\rangle} \boldsymbol{S}_{i} \cdot \boldsymbol{S}_{j}-\sum_{\langle i, j\rangle} \boldsymbol{D}_{i j} \cdot\left(\boldsymbol{S}_{i} \times \boldsymbol{S}_{j}\right)-d_{x} \sum_{i}\left(S_{i}^{x}\right)^{2}-d_{z} \sum_{i}\left(S_{i}^{z}\right)^{2} .
$$

We consider the nearest-neighbor exchange interaction, including an isotropic Heisenberg exchange $J$ and an antisymmetric DMI $\boldsymbol{D}_{i j}$, and a biaxial onsite-anisotropy with an easy axis $d_{z}$ in the $z$-direction and an intermediate axis $d_{x} \ll d_{z}$ along the $x$-direction, which specifies the favored plane of the DW as the $x z$-plane.
The time evolution of the spins is given by the stochastic Landau-Lifshitz-Gilbert equation (LLG), ${ }^{41}$

$$
-\frac{\left(1+\alpha^{2}\right) \mu_{s}}{\gamma} \frac{\partial}{\partial t} \boldsymbol{S}_{i}=\boldsymbol{S}_{i} \times \boldsymbol{H}_{i}+\alpha \boldsymbol{S}_{i} \times\left(\boldsymbol{S}_{i} \times \boldsymbol{H}_{i}\right)
$$

where $\gamma$ denotes the gyromagnetic ratio, $\mu_{s}$ the atomic magnetic moment, and $\alpha$ the Gilbert damping parameter. ${ }^{42}$ Within the framework of Langevin dynamics, temperature is included via an additional white noise term $\zeta_{i}(t)$ in the effective field,

$$
\boldsymbol{H}_{i}=-\frac{\partial \mathcal{H}}{\partial \boldsymbol{S}_{i}}+\zeta_{i}(t)
$$

with $\left\langle\zeta_{i}^{\eta}(t)\right\rangle=0$ and $\left\langle\zeta_{i}^{\eta}(t) \zeta_{j}^{v}\left(t^{\prime}\right)\right\rangle=2 \alpha k_{\mathrm{B}} T \frac{\mu_{s}}{\gamma} \delta_{i j} \delta^{\eta v} \delta\left(t-t^{\prime}\right){ }^{43}$

We solve the LLG equation numerically using Heun's method with time step $\Delta t=1.76 \times 10^{-4} \mu_{s}(\gamma|J|)^{-1}$ for a system elongated along the $z$-direction with a Néel-type DW initialized in the $x z$-plane. Absorbing boundary conditions in the form of enhanced Gilbert damping is applied along the $z$-direction, while the system is periodic along the other directions. ${ }^{21}$

For the investigation of the equilibrium properties of the static DW, the temperature was set constant for the whole system. To study the thermally induced dynamics, a linear temperature gradient $\frac{\Delta T}{\Delta z}$ was introduced along the longitudinal axis.

Position and profile of the DW were determined via the staggered (Néel) order parameter along the $z$-direction $2 \boldsymbol{n}(z)=\boldsymbol{m}_{1}(z)-\boldsymbol{m}_{2}(z)$, where $\boldsymbol{m}_{1 / 2}(z)$ are the magnetizations of the sublattices at position $z$ averaged over the $x y$-plane.

In real materials, DMI vectors never point only along the one axis. But since we are interested in a general study, not focusing on one material only, we decided to disentangle the variety of possible configurations of DWs and DMI vectors by modeling only DMI vectors along one axis at a time for three different configurations. With that we are able to demonstrate which component of (in reality much more complicated DMI vectors) makes a contribution to the static and dynamic behavior of DWs.

Hence, in the following, three different DMI configurations are systematically investigated: in the configuration $\mathrm{D}^{\mathrm{z}}$, the DMI vectors are aligned with the $z$-direction, the easy axis of the model. In the configuration $D^{\|}$, the DMI vectors are aligned with the $x$-axis, the axis with intermediate anisotropy. In the configuration $\mathrm{D}^{\perp}$, the vectors are aligned with the $y$-axis, the hard axis. A sketch of the investigated system and DMI configurations can be found in Fig. 1. As we will show in the following, the last two configurations change the equilibrium properties of the wall, while the first one breaks the symmetry of the dispersion relation.

\section{RESULTS}

\section{A. Static properties of the DW}

To understand the influence of the DMI configurations on the DW profile and energy and for comparison with our simulations, we start with analytical calculations within the framework of an one-dimensional micromagnetic model, where the antiferromagnetic order parameter $\boldsymbol{n}(z)$ is a continuous function of $z$. For this purpose, we express the energy density of the system described by 


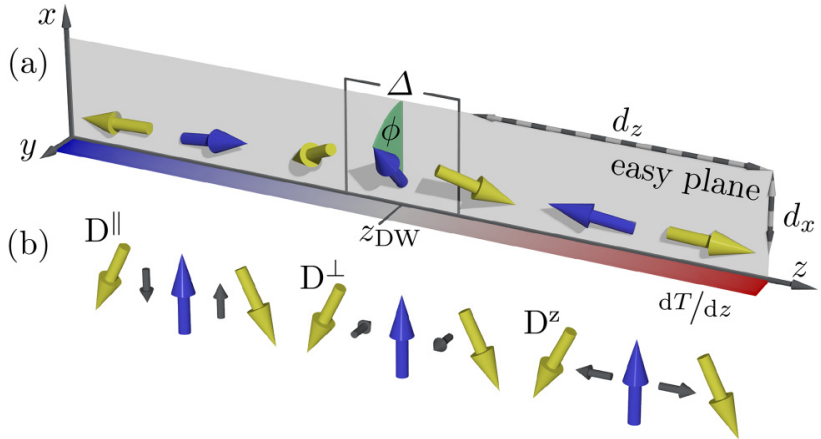

FIG. 1. (a) Sketch of an one-dimensional representation of the system. The spins from sublattice A (blue) and sublattice B (yellow) are represented by arrows. The easy $x z$-plane, created by our choice of anisotropy parameters, is represented by the gray plane and the linear temperature gradient $\frac{\Delta T}{\Delta z}>0$ by the color gradient in the $z$-direction. The resulting $D W$ is a Néel-head-to-head DW with position $z_{\mathrm{DW}}$, tilt angle $\phi$, and width $\Delta$. (b) Schematic illustration of the investigated DMI configurations. The black arrows indicate the DMI vectors.

the Hamiltonian in Eq. (1) in spherical coordinates,

$$
\begin{aligned}
\epsilon= & \int_{-\infty}^{\infty} \mathrm{d} z\left(w_{\text {exc }}+w_{\text {anis }}+w_{\mathrm{dmi}}\right) \\
= & \int_{-\infty}^{\infty} \mathrm{d} z\left[A \theta^{\prime 2}-D_{\mathrm{m}}^{x} \theta^{\prime} \sin \phi+D_{\mathrm{m}}^{y} \theta^{\prime} \cos \phi\right. \\
& \left.-K_{x} \cos ^{2} \phi \sin ^{2} \theta-K_{z} \cos ^{2} \theta\right]
\end{aligned}
$$

where $A=\frac{J}{2 a}$ is the exchange stiffness, $D_{\mathrm{m}}=\frac{D}{a^{2}}$ the micromagnetic DMI vector, and $K_{x}=\frac{d_{x}}{a^{3}}$ and $K_{z}=\frac{d_{z}}{a^{3}}$ the biaxial anisotropies in the continuum model.

$\phi(z)$ and $\theta(z)$ denote the angles between order parameter and $x$ - and $z$-axes. We assume $\phi(z)$ to be constant throughout the wall defining the global tilt angle of the DW. Similar calculations were carried out in Refs. 44-46, whereby in our work, the energy of the system is explicitly calculated as a continuous function of the tilt angle $\phi$.

The static DW profile $\boldsymbol{n}_{\mathrm{DW}}$ can be calculated by minimizing the energy density. Variation of $\theta(z)$ by solving the EulerLagrange equation, while meeting the boundary conditions $\lim _{z \rightarrow-\infty} \theta(z)=0$ and $\lim _{z \rightarrow+\infty} \theta(z)=\pi$ result in the well known DW profile,

$$
\begin{gathered}
n_{\mathrm{DW}}^{x}(z)=\frac{\cos \phi}{\cosh \left(\frac{z-z_{\mathrm{DW}}}{\Delta}\right)}, \\
n_{\mathrm{DW}}^{y}(z)=\frac{\sin \phi}{\cosh \left(\frac{z-z_{\mathrm{DW}}}{\Delta}\right)}, \\
n_{\mathrm{DW}}^{z}(z)=-\tanh \left(\frac{z-z_{\mathrm{DW}}}{\Delta}\right),
\end{gathered}
$$

with the DW width

$$
\Delta=\sqrt{A / K_{z}-K_{x} \cos ^{2} \phi} .
$$

At this point, it can be seen that the DMI does not enter explicitly in the DW profile, but-as we will show in the following-indirectly via the tilt angle $\phi$. The minimized energy density can be obtained by solving the integral in Eq. (4) after inserting Eq. (5) and reads as follows:

$$
\epsilon=4 \sqrt{A\left(K_{z}-K_{x} \cos ^{2} \phi\right)}-D_{\mathrm{m}}^{x} \pi \sin \phi+D_{\mathrm{m}}^{y} \pi \cos \phi
$$

This energy density depends on $\phi$ and only the $x$ - and $y$-components of the DMI vector $D_{\mathrm{m}}$. Hence, only the configurations $\mathrm{D}^{\|}$and $\mathrm{D}^{\perp}$ can play a role. Above a critical value of $D_{c}^{x}=\frac{4}{\pi} \sqrt{A K_{z}}$, respectively, $D_{c}^{y}=\frac{4}{\pi} \sqrt{A\left(K_{z}-K_{x}\right)}$, the energy density becomes negative and hence the DWs proliferate in the system resulting in a spin spiral state, which has been studied before. ${ }^{32,47,48}$ In our simulations, we always stay far below this critical point.

Additionally, the tilt angle $\phi$, which minimizes the energy density, is a function of the DMI. In the absence of the DMI, or for the $\mathrm{D}^{\mathrm{z}}$ configuration, two degenerate minima can be found at $\phi=0$ and $\phi=\pi$.

For the configuration $\mathrm{D}^{\|}$with $\boldsymbol{D}=D \boldsymbol{e}_{x}$, we obtain

$$
\phi(D)= \begin{cases}\operatorname{sgn} D \cdot \frac{\pi}{2} & \text { if }|D|>D^{\text {sat }} \\ \operatorname{sgn} D \cdot \arccos ( \pm \Pi) & \text { else }\end{cases}
$$

with the substitution

$$
\Pi=\sqrt{\frac{8 J d_{x}^{2}-D^{2} d_{z} \pi^{2}}{8 J d_{x}^{2}-D^{2} d_{x} \pi^{2}}} .
$$

At the DMI strength $D^{\text {sat }}=\frac{4}{\pi} \sqrt{\frac{J d_{x}^{2}}{2 d_{z}}}$, the effect of the DMI saturates. By inserting Eq. (8) into Eq. (6), the DW width is obtained as a function of $D$,

$$
\Delta(D)=\left\{\begin{array}{cl}
\sqrt{\frac{I}{2}\left[d_{z}-d_{x} \Pi^{2}\right]^{-1}} & \text { if }|D|<D^{\mathrm{sat}} \\
\sqrt{J\left(2 d_{z}\right)^{-1}} & \text { else. }
\end{array}\right.
$$

To compare these analytical results with our simulations, a system of $16 \times 16 \times 128$ spins with $\alpha=0.01, \quad d_{x}=0.02|J|$, $d_{z}=0.1|J|$, and $T=0.05|J| k_{\mathrm{B}}^{-1}$ was simulated. The DMI strength $D$ was varied from $D=-0.10|J|$ to $D=0.10|J|$ with a step size of $0.01|J|$. In all simulations, the DW was initialized with tilt angle $\phi=0$ in the easy plane. For all parameters, 20 runs with different seeds were simulated to allow for a statistical evaluation. By fitting the calculated DW profile $\boldsymbol{n}_{\mathrm{DW}}(z)$ [Eq. (5)] to the system's order parameter, the properties of the DW are determined. As can be seen in Fig. 2, we obtain perfect agreement between the analytical theory and the numerical data in spite of the completely different approaches.

For the configuration $\mathrm{D}^{\perp}$ with $\boldsymbol{D}=D \boldsymbol{e}_{y}$, the degeneracy of the two energy minima is lifted meaning that the DMI favors one 


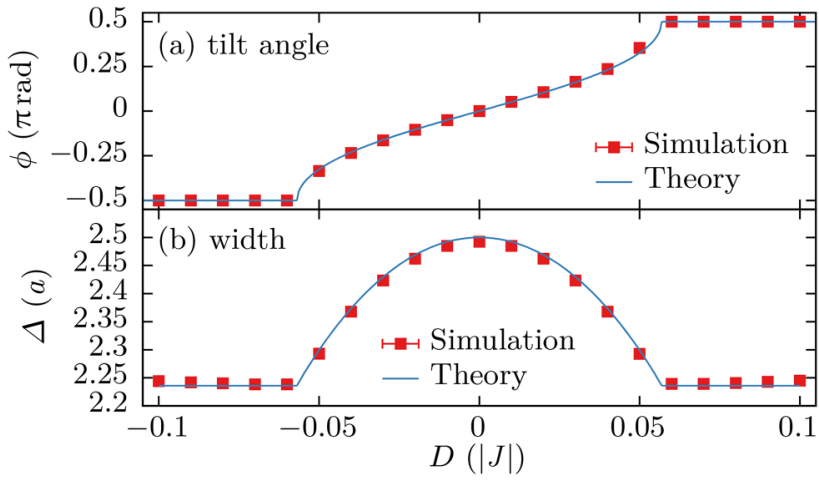

FIG. 2. (a) Tilt angle $\phi$ and (b) DW width $\Delta$ as functions of the DMI strength $D$. Analytical results [Eqs. (8) and (6)] and results from simulations are compared for the configuration $D^{\|}$

chirality of the DW. At a critical value of

$$
D^{\text {switch }}\left(d_{x}\right)=\frac{4 d_{x}}{\pi} \sqrt{\frac{J}{2\left(d_{z}-d_{x}\right)}},
$$

the minimum at $\phi=0$ vanishes and the wall flips. For the tilt angle, we obtain

$$
\phi(D)= \begin{cases}0 & \text { if } D<D^{\text {switch }} \\ \pi & \text { else. }\end{cases}
$$

This analytical result is also confirmed by our simulations and will be discussed in Sec. III B since it affects the dynamic behavior of the DW.

\section{B. Domain wall dynamics}

Domain wall dynamics in temperature gradients was introduced by Hinzke and Nowak ${ }^{19}$ and later by Schlickeiser et al. ${ }^{20}$ for FMs, and it was found that the motion of the DW is mainly caused by an entropic torque resulting from a temperature-dependent effective exchange interaction $A(T)$. Later on, the analogy to DWs in AFMs was investigated by Selzer et $a l^{26}$ In the following, we extend this work to the case of materials with DMI.

Within the same approach as used by Schlickeiser et al. ${ }^{20}$ and using the same linear approximation for the temperature dependence of the DMI, $\frac{\partial D_{m}(T)}{\partial T} \approx-\frac{2}{3} \frac{D_{m}(0)}{T_{\mathrm{N}}}$, as it was used for the exchange stiffness $\frac{\partial A(T)}{\partial T} \approx-\frac{2}{3} \frac{A(0)}{T_{\mathrm{N}}}$ in Ref. 26, we obtain the following formula for the DW velocity:

$$
v_{\mathrm{DW}}(D)=\frac{4 \gamma a^{2}}{3 \mu_{s} \alpha T_{\mathrm{N}}}\left(\frac{|J|}{2}-D \sin \eta(D)\right) \frac{\Delta T}{\Delta z} .
$$

Here, $T_{N}$ is the critical temperature and $a$ the atomic distance. The second term contains the contribution from DMI, which depends on the angle $\eta$ between the DMI vectors and the DW. For the case $\mathrm{D}^{\mathrm{z}}$, it is zero and no influence of the DMI on the DW dynamics can be expected. For the case $D^{\|}$, this angle can be calculated from Eq. (8), and it is $\eta=\phi$. For the case $\mathrm{D}^{\perp}$, this angle can be calculated from Eq. (12), and it is $\eta=\pi / 2+\phi$. As can be seen from Eq. (13), in the latter case, the DMI can speed-up or slow down the DW.

In order to investigate the influence of the DMI on the DW velocity $v_{\text {DW }}$ numerically, systems of size $16 \times 16 \times 128$ spins with temperature gradient $\frac{\Delta T}{\Delta z}=1.5 \times 10^{-3}|J|\left(k_{\mathrm{B}} a\right)^{-1}$ along the longitudinal axis, $d_{z}=0.1|J|, d_{x}=0.02|J|$, and $\alpha=0.01$ were simulated. The DMI strength was varied from $D=-0.1|J|$ to $D=0.1|J|$. In all simulations, the DW was initialized with tilt angle $\phi=0$. In order to have better statistics, all simulations were carried out 20 times with different seeds.

Figure 3 compares the analytical result [Eq. (13)] with the simulation results for the configurations $\mathrm{D}^{\|}$and $\mathrm{D}^{\perp}$. We obtain good agreement between the analytical theory and the numerical results. For the configuration $\mathrm{D}^{\|}$, the velocity of the $\mathrm{DW}$ is always reduced by the DMI. For the configuration $\mathrm{D}^{\perp}$, the situation is more complicated: if the wall is in the stable state with the energetically favorable chirality, the DW velocity is reduced. However, if the wall is in the meta-stable state characterized by the energetically unfavorable chirality, the velocity increases. This can only be observed in the configuration $\mathrm{D}^{\perp}$ with $0<D<D^{\text {switch }}$. The sketch in Fig. 4 illustrates that in this configuration the torques driving the DW can either support or hinder the torques from the isotropic exchange, leading to either an increasing or decreasing DW velocity depending on the sign of $D$.

The systematic deviations from the analytical theory in Fig. 3 can be explained by the fact that the thermal DMI field also entails canting of the sublattices in the DW, which leads to additional torques on the DW that intensify the effect. ${ }^{27}$ Despite the effect of the DMI on the equilibrium tilt angle, there is no Walker breakdown, as usual for DW in AFM. ${ }^{26}$

\section{Asymmetry of the dispersion relation}

On a microscopic level, the effective decrease in the exchange stiffness results from the population of magnons and the consequent reduction of the magnetization. The higher the temperature, the more

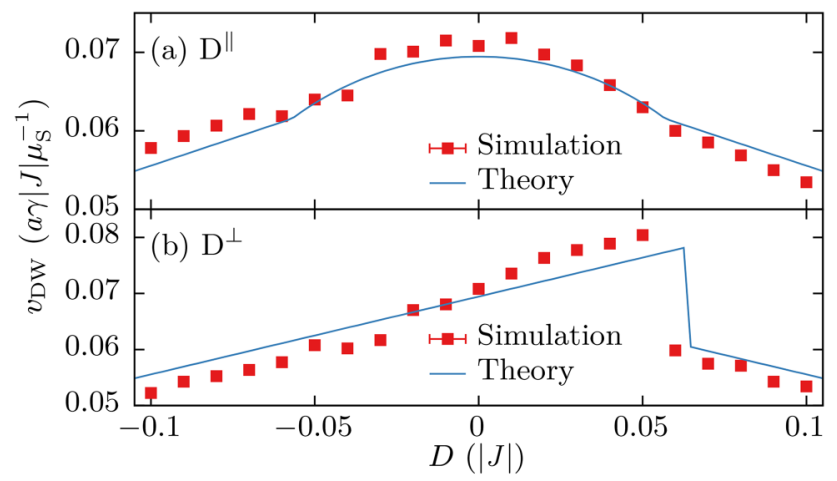

FIG. 3. DW velocity $v_{D W}$ as a function of the DMI strength $D$ for DMI configuration: (a) $D^{\|}$and (b) $D^{\perp}$. Analytical results [Eq. (13)] and results from simulations are compared. 


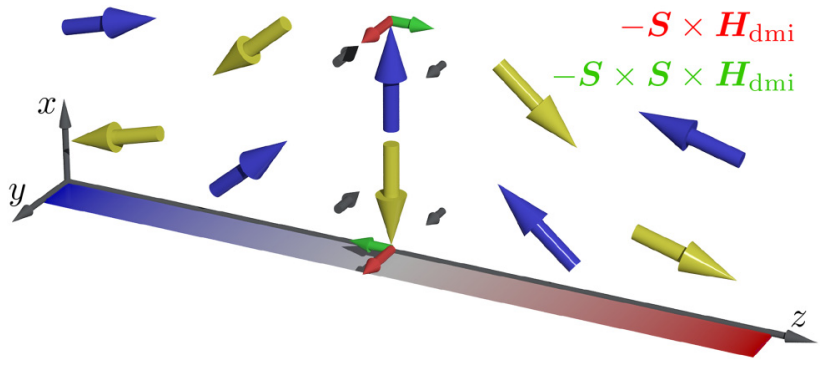

FIG. 4. Sketch of the DMI torques acting on a DW in a temperature gradient for the configuration $D^{\perp}$. The DMI vectors are depicted in black. The two sublattices of the AFM are indicated with blue and yellow spins. Depending on the sign of $D$, the green torques might support or hinder the corresponding torques of the isotropic exchange (not shown) that drive the DW motion.

magnons are excited. Hence, in a temperature gradient, a net current of magnons travels from the hot to the cold end driving the DW. ${ }^{19,21,49}$

Without DMI, the dispersion relation is symmetric in $\boldsymbol{q}$, which means that the mean magnonic propagation length $\bar{\xi}$ and the resulting magnon current are symmetric. In a FM with DMI this symmetry is broken, ${ }^{39,40}$ potentially leading to different DW velocities in antiparallel directions. ${ }^{38}$

For a deeper understanding of this asymmetry in AFMs, we start with a calculation of the mean magnon propagation length. As a first step, we calculate the propagation length $\xi(\boldsymbol{q})$ as a function of the wave vector $\boldsymbol{q}$. For that we solve the LLG [Eq. (2)] with damping term and DMI within linear spin wave theory, which leads to the dispersion relation $\omega_{\sigma}(\boldsymbol{q})$ and the inverse lifetime $\Gamma(\boldsymbol{q})$, where $\sigma$ indicates the two branches of the dispersion relation. The first result is that only the $z$-component of the DMI vector $\boldsymbol{D}$ appears in these two quantities, which means that only the DMI configuration $\mathrm{D}^{\mathrm{z}}$ can play a role. With these quantities, the propagation length $\xi(\boldsymbol{q})=\left|\frac{\partial \omega_{\sigma}}{\partial \boldsymbol{q}}\right| \Gamma^{-1}$ can be calculated and reads

$$
\begin{aligned}
\xi(\boldsymbol{q})= & \frac{\left(1+\alpha^{2}\right)}{\alpha} \frac{2 a \tilde{\Omega}_{q}}{\tilde{\Omega}_{0} \sqrt{\tilde{\Omega}_{0}^{2}-\left(1+\alpha^{2}\right) \tilde{\Omega}_{q}^{2}}} \\
& \cdot \sqrt{\sum_{\beta}\left[|J| \sin \left(q^{\beta} a\right)-\left|D_{\beta}\right| \cos \left(q^{\beta} a\right)\right]^{2}},
\end{aligned}
$$

whereby the substitutions $\tilde{J}_{\boldsymbol{q}}=2|J| \sum_{\beta} \cos \left(q^{\beta} a\right), \quad \tilde{D}_{\boldsymbol{q}}=2 \sum_{\beta}\left|D_{\beta}\right|$ $\times \sin \left(q^{\beta} a\right), \tilde{\Omega}_{0}=\tilde{J}_{0}+2 d_{z}$, and $\tilde{\Omega}_{q}=\tilde{J}_{q}+\tilde{D}_{q}$ are used. With $|D|>0$, the function is no longer symmetric with respect to $q=0$ but shifted.

To determine the mean propagation lengths $\bar{\xi}_{-}\left(\bar{\xi}_{+}\right)$of left (right) propagating magnons with negative (positive) group velocity $v_{\mathrm{g}}^{z}$, the integral

$$
\begin{aligned}
\bar{\xi}_{ \pm}= & \frac{V}{N} \frac{1}{(2 \pi)^{3}} \sum_{\sigma} \iiint_{\mathrm{BZ}} \mathrm{d} \boldsymbol{q} \frac{v_{\mathrm{g}, \sigma}^{z}(\boldsymbol{q})}{\Gamma(\boldsymbol{q})} \frac{k_{\mathrm{B}} T}{\omega_{\sigma}(\boldsymbol{q})} \\
& \exp \left(\frac{-2 \Delta z}{\xi_{\sigma}(\boldsymbol{q}) \cos \vartheta(\boldsymbol{q})}\right) \Theta\left( \pm v_{\mathrm{g}, \sigma}^{z}(\boldsymbol{q})\right)
\end{aligned}
$$

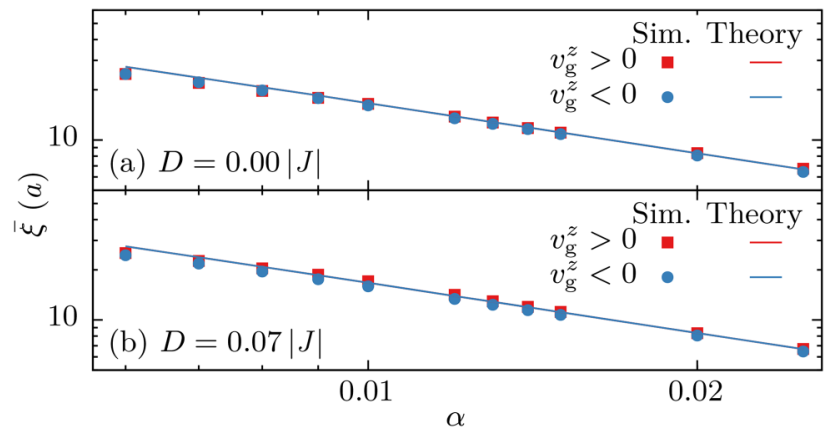

FIG. 5. Mean propagation length $\bar{\xi}$ in antiferromagnets as a function of the damping constant $\alpha$ for DMI configuration $\mathrm{D}^{\mathrm{z}}$ with strength (a) $D=0$ and (b) $D=0.07|J|$. The results from the analytical theory are compared with the results from simulations.

has to be solved numerically (see also Ref. 21). In the integral, the propagation length in the $z$-direction $\frac{v_{\mathrm{g}, \sigma}^{z}(\boldsymbol{q})}{\Gamma(\boldsymbol{q})}$ is averaged over the first Brillouin zone (BZ), whereby each mode $\boldsymbol{q}$ is weighted with its occupation probability $\frac{k_{\mathrm{B}} T}{a(\boldsymbol{q}) \omega_{\sigma}(\boldsymbol{q})}$ and amplitude $a(\boldsymbol{q})$. The exponential function corrects the fact that the modes can also propagate with angle $\vartheta$ with respect to the $z$-direction. With the Heaviside step function $\Theta$, the left and right propagating modes are evaluated separately.

To compare the results from this analytical theory with simulations, we simulate a system of $16 \times 16 \times 1024$ spins with a temperature step from $T=0$ to $T=0.2|J| k_{\mathrm{B}}^{-1}$ with $d_{z}=0.1|J|$ and $d_{x}=0$. We determine the magnon accumulation $\Delta \boldsymbol{n}=\left|\boldsymbol{n}_{0}(z)-\boldsymbol{n}(z)\right|$ in the vicinity of a temperature step. From the exponential decay of this accumulation $\Delta n^{z}(z)=\Delta n^{z}(0) \exp \frac{z}{\bar{E}}$, the propagation length can be obtained via fitting. ${ }^{50}$ The results are shown in Fig. 5.

Interestingly, in contrast to the case of a FM, ${ }^{40}$ the DMI does not break the symmetry of the mean propagation length. The reason for this is that the effect of the DMI on the two magnon branches has opposite sign and hence cancels out because both magnon branches are excited equally.

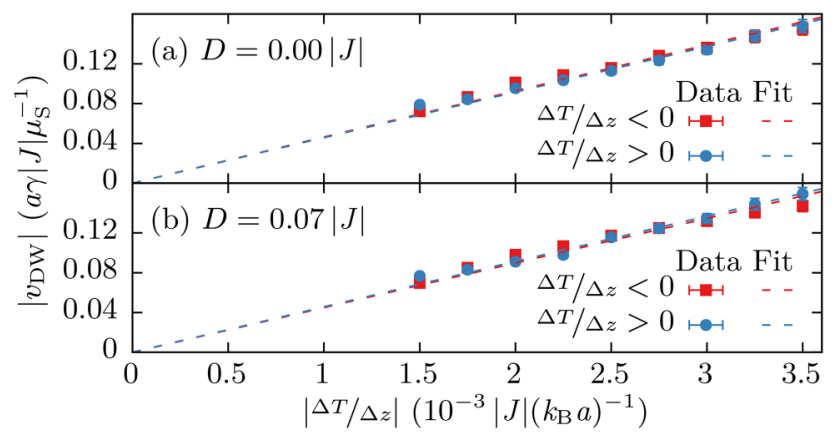

FIG. 6. Magnitude of DW velocity $\left|v_{D W}\right|$ as a function of the magnitude of temperature gradient $\left|\frac{\Delta T}{\Delta z}\right|$ for DMl configuration $D^{2}$ with DMl strength (a) $D=0.00|\mathrm{~J}|$ and (b) $D=0.07|\mathrm{~J}|$. Data points from simulations and linear fits. 
With this result, the spatial symmetry of the DW movement in AFMs should also be unaffected by the DMI. To verify this, we simulate a system of $16 \times 16 \times 128$ spins with a damping constant $\alpha=0.01$, the easy axis $d_{z}=0.1|J|$, and the intermediate axis $d_{x}=0.02|J|$. Temperature gradients were applied along the $z$-axis, each with positive and negative signs. Figure 6 shows the DW velocity for $D=0$ and $D=0.07|J|$. As expected from the theory, the DMI has no effect on the symmetry of the DW motion in AFMs.

\section{SUMMARY}

In this work, we investigate the influence of DMI on the static as well as dynamic properties of thermally driven DWs in AFMs. In the configuration $D^{\|}$, where the DMI vectors are aligned with the axis of intermediate anisotropy, the angle of the plane containing the DW is tilted leading to a reduced DW width and to additional torques that slow down the DW motion.

In the configuration $\mathrm{D}^{\perp}$, the vectors are aligned with the hard axis. Here, the DMI favors a certain chirality of the DW. The DW velocity can be reduced or enhanced depending on the DW chirality.

For the DMI configuration $\mathrm{D}^{\mathrm{z}}$, where the DMI vectors are aligned with the easy axis of the model, the static DW properties are unaffected. Here, an asymmetric dispersion relation can be observed which-for the case of FMs - can lead to different DW velocities in antiparallel directions. ${ }^{38}$ In AFMs, this effect is canceled by the symmetry of the sublattices. However, in all configurations, the symmetry of the torques acting on the two sublattices is preserved and, thus, there is no Walker breakdown. ${ }^{26}$

\section{ACKNOWLEDGMENTS}

This work was financially supported by the Deutsche Forschungsgemeinschaft through the Sonderforschungsbereich 767 at the University of Konstanz and through Project No. 290/7-1.

\section{DATA AVAILABILITY}

The data that support the findings of this study are available from the corresponding author upon reasonable request.

\section{REFERENCES}

1J. Nogués and I. K. Schuller, J. Magn. Magn. Mater. 192, 203 (1999).

2I. K. Schuller, R. Morales, X. Batlle, U. Nowak, and G. Güntherodt, J. Magn. Magn. Mater. 416, 2 (2016).

${ }^{3}$ B. G. Park, J. Wunderlich, X. Martí, V. V. Holý, Y. Kurosaki, M. Yamada, H. Yamamoto, A. Nishide, J. Hayakawa, H. Takahashi et al., Nat. Mater. 10, 347 (2011).

${ }^{4}$ T. Jungwirth, X. Marti, P. Wadley, and J. Wunderlich, Nat. Nanotechnol. 11, 231 (2016).

${ }^{\mathbf{5}}$ O. Gomonay, T. Jungwirth, and J. Sinova, Phys. Status Solidi Rapid Res. Lett. 11, 1700022 (2017).

${ }^{6}$ V. Baltz, A. Manchon, M. Tsoi, T. Moriyama, T. Ono, and Y. Tserkovnyak, Rev. Mod. Phys. 90, 015005 (2018).

${ }^{7}$ A. H. MacDonald and M. Tsoi, Philos. Trans. R. Soc. A 369, 3098 (2011).

${ }^{8}$ J. Železný, P. Wadley, K. Olejník, A. Hoffmann, and H. Ohno, Nat. Phys. 14, 220 (2018).

${ }^{\mathbf{9}}$ O. Gomonay, T. Jungwirth, and J. Sinova, Phys. Rev. Lett. 117, 017202 (2016).
${ }^{10}$ N. Thielemann-Kühn, D. Schick, N. Pontius, C. Trabant, R. Mitzner, K. Holldack, H. Zabel, A. Föhlisch, and C. Schüßler-Langeheine, Phys. Rev. Lett. 119, 197202 (2017).

${ }^{11}$ K. Olejník, T. Seifert, Z. Kašpar, V. Novák, P. Wadley, R. P. Campion, M. Baumgartner, P. Gambardella, P. Němec, J. Wunderlich et al., Sci. Adv. 4, eaar3566 (2018).

${ }^{12}$ L. Rózsa, S. Selzer, T. Birk, U. Atxitia, and U. Nowak, Phys. Rev. B 100, 064422 (2019).

${ }^{13}$ S. S. P. Parkin, M. Hayashi, and L. Thomas, Science 320, 190 (2008).

${ }^{14}$ N. L. Schryer and L. R. Walker, J. Appl. Phys. 45, 5406 (1974).

${ }^{15}$ G. S. D. Beach, C. Nistor, C. Knutson, M. Tsoi, and J. L. Erskine, Nat. Mater. 4, 741 (2005).

${ }^{16}$ G. Tatara and H. Kohno, Phys. Rev. Lett. 92, 086601 (2004).

${ }^{17}$ A. Yamaguchi, T. Ono, S. Nasu, K. Miyake, K. Mibu, and T. Shinjo, Phys. Rev. Lett. 92, 077205 (2004).

${ }^{18}$ A. Thiaville, Y. Nakatani, J. Miltat, and Y. Suzuki, Europhys. Lett. 69, 990 (2005).

${ }^{19}$ D. Hinzke and U. Nowak, Phys. Rev. Lett. 107, 027205 (2011).

${ }^{20}$ F. Schlickeiser, U. Ritzmann, D. Hinzke, and U. Nowak, Phys. Rev. Lett. 113, 097201 (2014).

${ }^{\mathbf{2 1}}$ A. Donges, N. Grimm, F. Jakobs, S. Selzer, U. Ritzmann, U. Atxitia, and U. Nowak, Phys. Rev. Res. 2, 013293 (2020).

${ }^{22}$ Y. A. Shokr, O. Sandig, M. Erkovan, B. Zhang, M. Bernien, A. A. Ünal, F. Kronast, U. Parlak, J. Vogel, and W. Kuch, Phys. Rev. B 99, 214404 (2019).

${ }^{23}$ M. Bode, E. Y. Vedmedenko, K. V. Bergmann, A. Kubetzka, P. Ferriani, S. Heinze, and R. Wiesendanger, Nat. Mater. 5, 477 (2006).

${ }^{24}$ E. G. Tveten, A. Qaiumzadeh, and A. Brataas, Phys. Rev. Lett. 112, 147204 (2014).

${ }^{\mathbf{2 5}}$ S. K. Kim, Y. Tserkovnyak, and O. Tchernyshyov, Phys. Rev. B 90, 104406 (2014), arXiv:1406.6051.

${ }^{\mathbf{2 6}}$ S. Selzer, U. Atxitia, U. Ritzmann, D. Hinzke, and U. Nowak, Phys. Rev. Lett. 117, 107201 (2016).

${ }^{27}$ S.-H. Yang, K.-S. Ryu, and S. Parkin, Nat. Nanotechnol. 10, 221 (2015).

${ }^{28}$ M. Kuteifan, M. V. Lubarda, S. Fu, R. Chang, M. A. Escobar, S. Mangin, E. E. Fullerton, and V. Lomakin, AIP Adv. 6, 045103 (2016).

${ }^{29}$ I. Dzyaloshinsky, J. Phys. Chem. Solids 4, 241 (1958).

${ }^{30}$ T. Moriya, Phys. Rev. Lett. 4, 228 (1960).

${ }^{31}$ T. Moriya, Phys. Rev. 120, 91 (1960).

${ }^{32}$ M. Bode, M. Heide, K. V. Bergmann, P. Ferriani, S. Heinze, G. Bihlmayer, A. Kubetzka, O. Pietzsch, S. Blügel, and R. Wiesendanger, Nature 447, 190 (2007).

${ }^{33}$ U. K. Rößler, A. N. Bogdanov, and C. Pfleiderer, Nature 442, 797 (2006).

${ }^{34}$ S. Mühlbauer, B. Binz, F. Jonietz, C. Pfleiderer, A. Rosch, A. Neubauer, R. Georgii, and P. Böni, Science 323, 915 (2009).

${ }^{35}$ X. Z. Yu, N. Kanazawa, Y. Onose, K. Kimoto, W. Z. Zhang, S. Ishiwata, Y. Matsui, and Y. Tokura, Nat. Mater. 10, 106 (2011).

${ }^{36}$ A. Thiaville, S. Rohart, É. Jué, V. Cros, and A. Fert, Europhys. Lett. 100, 57002 (2012).

${ }^{37}$ W. Wang, M. Albert, M. Beg, M.-A. Bisotti, D. Chernyshenko, D. Cortés-Ortuño, I. Hawke, and H. Fangohr, Phys. Rev. Lett. 114, 087203 (2015).

${ }^{38}$ S.-G. Je, D.-H. Kim, S.-C. Yoo, B.-C. Min, K.-J. Lee, and S.-B. Choe, Phys. Rev. B 88, 214401 (2013).

${ }^{39}$ K. Zakeri, Y. Zhang, J. Prokop, T.-H. Chuang, N. Sakr, W. X. Tang, and J. Kirschner, Phys. Rev. Lett. 104, 137203 (2010).

${ }^{40}$ J.-H. Moon, S.-M. Seo, K.-J. Lee, K.-W. Kim, J. Ryu, H.-W. Lee, R. D. McMichael, and M. D. Stiles, Phys. Rev. B 88, 184404 (2013).

${ }^{41}$ L. Landau and E. Lifshits, Phys. Z. Sow. 8, 153-169 (1935); see also http:// archive.ujp.bitp.kiev.ua/index.php?item=j\&id=110.

${ }^{42}$ T. L. Gilbert, IEEE Trans. Magn. 40, 3443 (2004).

${ }^{43}$ W. F. Brown, Phys. Rev. 130, 1677 (1963).

${ }^{44}$ B. Schweflinghaus, B. Zimmermann, M. Heide, G. Bihlmayer, and S. Blügel, Phys. Rev. B 94, 024403 (2016). 
${ }^{45} \mathrm{M}$. Heide, G. Bihlmayer, and S. Blügel, Phys. Rev. B 78, 140403 (2008).

${ }^{46}$ S. Rohart and A. Thiaville, Phys. Rev. B 88, 184422 (2013).

${ }^{47}$ P. Ferriani, K. von Bergmann, E. Y. Vedmedenko, S. Heinze, M. Bode, M. Heide, G. Bihlmayer, S. Blügel, and R. Wiesendanger, Phys. Rev. Lett. 101, 027201 (2008).
${ }^{48}$ G. Hasselberg, R. Yanes, D. Hinzke, P. Sessi, M. Bode, L. Szunyogh, and U. Nowak, Phys. Rev. B 91, 064402 (2015).

${ }^{49}$ P. Yan, X. S. Wang, and X. R. Wang, Phys. Rev. Lett. 107, 177207 (2011).

${ }^{50}$ U. Ritzmann, D. Hinzke, and U. Nowak, Phys. Rev. B 89, 024409 (2014). 